\title{
Pisa syndrome after rasagiline therapy in a patient with Parkinson's disease
}

\author{
F. Valentino ${ }^{1} \cdot$ G. Cosentino $^{1} \cdot$ B. Fierro $^{1} \cdot$ S. Realmuto $^{1} \cdot$ S. Mastrilli $^{1}$ \\ G. Savettieri' ${ }^{1}$ M. D'Amelio ${ }^{1}$
}

Received: 17 May 2015/Accepted: 21 August 2015/Published online: 3 September 2015

(C) Springer-Verlag Italia 2015

\section{Dear Editor,}

We have read with interest comments by Solla et al. [1] regarding our Letter to the Editor published in Neurological Sciences titled "Insidious onset of Pisa Syndrome after rasagiline therapy in a patient with Parkinson's Disease" [2]. We thank the authors for their interest in our paper, but we think it is necessary to make some clarifications regarding the temporal relationship between the onset of Pisa Syndrome (PS) and rasagiline therapy. PS occurred in fact after and not before rasagiline treatment as stated by Solla et al. In particular, PS occurred after a time interval of 6-12 months and anyway after rasagiline was added to patient's therapy. This long time interval is one of the elements that makes the case interesting (time period longer than previously described). Temporal relationship is fundamental for the interpretation of clinical and electrophysiological findings in our case.

Rasagiline is an inhibitor of monoamine oxidase type B, and although different mechanisms of action have been advocated, its effect is primarily dopamine mediated [3]. This means that occurrence of Pisa syndrome in our case was likely related not only to the specific mechanisms of action of the drug but also to increased dopamine extracellular levels at the striatal synapses, and thus to the total dopamine agonism load.

To conclude, though it is likely that concomitant use of other antiparkinsonian drugs could have contributed to the onset of PS in our patient, it is noteworthy that PS was not evident before rasagiline was started, it became evident after rasagiline was initiated, and it was not anymore evident after rasagiline was stopped.

\section{Compliance with ethical standards}

Ethical standards All procedures were conducted according to the ethical standards of studies involving human subjects (declaration of Helsinki).

Conflict of interest The authors declare that they have no financial or other conflicts of interest in relation to this research and its publication.

\section{References}

1. Solla P, Cannas A, Orofino G, Marrosu F (2015) Rasagiline and Pisa syndrome in Parkinson's disease patients. Neurol Sci 36(3):485-486

2. Valentino F, Cosentino G, Fierro B, Realmuto S, Mastrilli S, Savettieri G, D'Amelio M (2014) Insidious onset of Pisa syndrome after rasagiline therapy in a patient with Parkinson's disease. Neurol Sci 35(10):1615-1617

3. Weinreb O, Amit T, Bar-Am O, Youdim MB (2010) Rasagiline: a novel anti-Parkinsonian monoamine oxidase-B inhibitor with neuroprotective activity. Prog Neurobiol 92(3):330-344
M. D'Amelio

marco.damelio@unipa.it

1 Dipartimento di Biomedicina Sperimentale e Neuroscienze Cliniche, Università degli Studi di Palermo, Via Gaetano La Loggia 1, 90129 Palermo, Italy 